\title{
Some Preliminary Results about Uncertain Matrix
}

Baoding Liu

Correspondence:

liu@tsinghua.edu.cn; http://orsc.edu.cn/liu

Department of Mathematical Sciences, Tsinghua University, Beijing 100084, China

\begin{abstract}
This paper presents a new concept of uncertain matrix that is a measurable function from an uncertainty space to the set of real matrices. It is proved that an uncertain matrix is a matrix all of whose elements are uncertain variables. The independence of uncertain matrices is also investigated.
\end{abstract}

Keywords: Uncertainty theory; Uncertain measure; Uncertain matrix

\section{Introduction}

In order to rationally model belief degrees, uncertainty theory was founded by Liu [1] in 2007 and perfected by Liu [2] in 2009 with the fundamental concept of uncertain measure. Following that, uncertainty theory has been developed steadily and applied in science and engineering.

Roughly speaking, an uncertain element (Liu [1]) is a measurable function from an uncertainty space to a collection of some objects. The uncertain element is an uncertain variable when the collection consists of real numbers, an uncertain vector when the collection consists of real vectors, an uncertain sequence when the collection consists of infinite-dimensional real vector, an uncertain process (Liu [3]) when the collection consists of functions of time or space, and an uncertain set (Liu [4]) when the collection consists of sets of real numbers.

As a new subtopic of uncertain element, this paper will present a concept of uncertain matrix that is a measurable function from an uncertainty space to the set of real matrices. We will prove that an uncertain matrix is a matrix all of whose elements are uncertain variables. The independence of uncertain matrices will also be investigated.

\section{Preliminaries}

Let $\Gamma$ be a nonempty set and $\mathcal{L}$ a $\sigma$-algebra over $\Gamma$. Each element $\Lambda$ in $\mathcal{L}$ is called an event. Liu [1] defined an uncertain measure by the following axioms:

Axiom 1. (Normality axiom) $\mathcal{M}\{\Gamma\}=1$ for the universal set $\Gamma$;

Axiom 2. (Duality axiom) $\mathcal{M}\{\Lambda\}+\mathcal{M}\left\{\Lambda^{c}\right\}=1$ for any event $\Lambda$;

Axiom 3. (Subadditivity axiom) For every countable sequence of events $\Lambda_{1}, \Lambda_{2}, \cdots$, we have

$$
\mathcal{M}\left\{\bigcup_{i=1}^{\infty} \Lambda_{i}\right\} \leq \sum_{i=1}^{\infty} \mathcal{M}\left\{\Lambda_{i}\right\} .
$$

(c) The Author(s). 2016 Open Access This article is distributed under the terms of the Creative Commons Attribution 4.0 International License (http://creativecommons.org/licenses/by/4.0/), which permits unrestricted use, distribution, and reproduction in any medium, provided you give appropriate credit to the original author(s) and the source, provide a link to the Creative Commons license, and indicate if changes were made. 
The triplet $(\Gamma, \mathcal{L}, \mathcal{M})$ is called an uncertainty space. Furthermore, Liu [2] defined a product uncertain measure by the fourth axiom:

Axiom 4. (Product axiom) Let $\left(\Gamma_{k}, \mathcal{L}_{k}, \mathcal{M}_{k}\right)$ be uncertainty spaces for $k=1,2, \cdots$ The product uncertain measure $\mathcal{M}$ is an uncertain measure satisfying

$$
\mathcal{M}\left\{\prod_{k=1}^{\infty} \Lambda_{k}\right\}=\bigwedge_{k=1}^{\infty} \mathcal{M}_{k}\left\{\Lambda_{k}\right\}
$$

where $\Lambda_{k}$ are arbitrarily chosen events from $\mathcal{L}_{k}$ for $k=1,2, \cdots$, respectively.

An uncertain variable is defined by Liu [1] as a function $\xi$ from an uncertainty space $(\Gamma, \mathcal{L}, \mathcal{M})$ to the set of real numbers such that $\{\xi \in B\}$ is an event for any Borel set $B$. In order to describe an uncertain variable in practice, uncertainty distribution is defined by Liu [1] as

$$
\Phi(x)=\mathcal{M}\{\xi \leq x\}, \quad \forall x \in \Re .
$$

Peng and Iwamura [5] verified that a function $\Phi: \Re \rightarrow[0,1]$ is an uncertainty distribution if and only if it is a monotone increasing function except $\Phi(x) \equiv 0$ and $\Phi(x) \equiv 1$. An uncertainty distribution $\Phi(x)$ is said to be regular if it is a continuous and strictly increasing function with respect to $x$ at which $0<\Phi(x)<1$, and

$$
\lim _{x \rightarrow-\infty} \Phi(x)=0, \quad \lim _{x \rightarrow+\infty} \Phi(x)=1 .
$$

Let $\xi$ be an uncertain variable with regular uncertainty distribution $\Phi(x)$. Then, the inverse function $\Phi^{-1}(\alpha)$ is called the inverse uncertainty distribution of $\xi$ (Liu [6]). It is also verified that a function $\Phi^{-1}(\alpha):(0,1) \rightarrow \Re$ is an inverse uncertainty distribution if and only if it is a continuous and strictly increasing function with respect to $\alpha$. Independence is an extremely important concept in uncertainty theory. The uncertain variables $\xi_{1}, \xi_{2}, \ldots, \xi_{n}$ are said to be independent (Liu [2]) if

$$
\mathcal{M}\left\{\bigcap_{i=1}^{n}\left(\xi_{i} \in B_{i}\right)\right\}=\bigwedge_{i=1}^{n} \mathcal{M}\left\{\xi_{i} \in B_{i}\right\}
$$

for any Borel sets $B_{1}, B_{2}, \cdots, B_{n}$ of real numbers. Equivalently, those uncertain variables are independent if and only if

$$
\mathcal{M}\left\{\bigcup_{i=1}^{n}\left(\xi_{i} \in B_{i}\right)\right\}=\bigvee_{i=1}^{n} \mathcal{M}\left\{\xi_{i} \in B_{i}\right\}
$$

A $k$-dimensional uncertain vector (Liu [1]) is a function $\xi$ from an uncertainty space $(\Gamma, \mathcal{L}, \mathcal{M})$ to the set of $k$-dimensional real vectors such that $\{\xi \in B\}$ is an event for any Borel set $B$ of $k$-dimensional real vectors. It is showed that the vector $\left(\xi_{1}, \xi_{2}, \ldots, \xi_{k}\right)$ is an uncertain vector if and only if $\xi_{1}, \xi_{2}, \cdots, \xi_{k}$ are uncertain variables. The $k$-dimensional uncertain vectors $\xi_{1}, \xi_{2}, \cdots, \xi_{n}$ are said to be independent (Liu [7]) if for any Borel sets $B_{1}, B_{2}, \cdots, B_{n}$ of $k$-dimensional real vectors, we have

$$
\mathcal{M}\left\{\bigcap_{i=1}^{n}\left(\xi_{i} \in B_{i}\right)\right\}=\bigwedge_{i=1}^{n} \mathcal{M}\left\{\xi_{i} \in B_{i}\right\} .
$$

It is proved that the $k$-dimensional uncertain vectors $\xi_{1}, \xi_{2}, \cdots, \xi_{n}$ are independent if and only if

$$
\mathcal{M}\left\{\bigcup_{i=1}^{n}\left(\xi_{i} \in B_{i}\right)\right\}=\bigvee_{i=1}^{n} \mathcal{M}\left\{\xi_{i} \in B_{i}\right\}
$$


for any Borel sets $B_{1}, B_{2}, \cdots, B_{n}$ of $k$-dimensional real vectors.

\section{Uncertain Matrix}

This section introduces a new concept of uncertain matrix and proves that an uncertain matrix is a matrix all of whose elements are uncertain variables.

Definition $1 A p \times q$ uncertain matrix is a function $\xi$ from an uncertainty space $(\Gamma, \mathcal{L}, \mathcal{M})$ to the set of $p \times q$ real matrices such that $\{\xi \in B\}$ is an event for any Borel set $B$ of $p \times q$ real matrices.

Theorem 1 The $p \times q$ matrix $\xi$ is an uncertain matrix if and only if

$$
\xi=\left(\begin{array}{cccc}
\xi_{11} & \xi_{12} & \cdots & \xi_{1 q} \\
\xi_{21} & \xi_{22} & \cdots & \xi_{2 q} \\
\vdots & \vdots & \ddots & \vdots \\
\xi_{p 1} & \xi_{p 2} & \cdots & \xi_{p q}
\end{array}\right)
$$

where $\xi_{i j}, i=1,2, \cdots, p, j=1,2, \cdots, q$ are uncertain variables.

Proof Suppose that $\xi$ is defined on the uncertainty space $(\Gamma, \mathcal{L}, \mathcal{M})$. For any Borel set $B$ of real numbers, the set

$$
B^{*}=\left(\begin{array}{cccc}
B & \mathfrak{R} & \ldots & \mathfrak{R} \\
\mathfrak{R} & \mathfrak{R} & \ldots & \mathfrak{R} \\
\vdots & \vdots & \ddots & \vdots \\
\mathfrak{R} & \mathfrak{R} & \ldots & \mathfrak{R}
\end{array}\right)
$$

is a Borel set of $p \times q$ real matrices. Thus, the set $\left\{\xi_{11} \in B\right\}=\left\{\xi \in B^{*}\right\}$ is an event. Hence, $\xi_{11}$ is an uncertain variable. A similar process may prove that other $\xi_{i j} \mathrm{~s}$ are uncertain variables.

Conversely, suppose that all $\xi_{i j}$ s are uncertain variables on the uncertainty space $(\Gamma, \mathcal{L}, \mathcal{M})$. We define

$$
\mathcal{B}=\left\{B \subset \Re^{p \times q} \mid\{\xi \in B\} \text { is an event }\right\} .
$$

The matrix $\xi=\left(\xi_{i j}\right)_{p \times q}$ is proved to be an uncertain matrix if we can prove that $\mathcal{B}$ contains all Borel sets of $p \times q$ real matrices. First, the class $\mathcal{B}$ contains all open intervals of $\Re^{p \times q}$ because

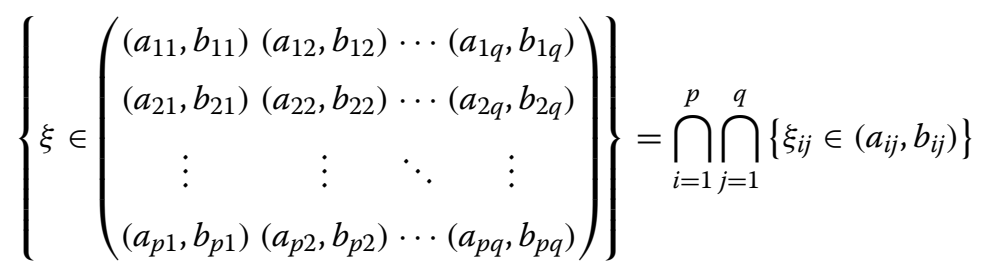

is an event. Next, the class $\mathcal{B}$ is a $\sigma$-algebra over $\Re^{p \times q}$ because (i) we have $\Re^{p \times q} \in \mathcal{B}$ since $\left\{\xi \in \mathfrak{R}^{p \times q}\right\}=\Gamma$; (ii) if $B \in \mathcal{B}$, then $\{\xi \in B\}$ is an event, and

$$
\left\{\xi \in B^{c}\right\}=\{\xi \in B\}^{c}
$$


is an event. This means that $B^{c} \in \mathcal{B}$; (iii) if $B_{i} \in \mathcal{B}$ for $i=1,2, \cdots$, then $\left\{\xi \in B_{i}\right\}$ are events and

$$
\left\{\xi \in \bigcup_{i=1}^{\infty} B_{i}\right\}=\bigcup_{i=1}^{\infty}\left\{\xi \in B_{i}\right\}
$$

is an event. This means that $\cup_{i} B_{i} \in \mathcal{B}$. Since the smallest $\sigma$-algebra containing all open intervals of $\Re^{p \times q}$ is just the Borel algebra over $\Re^{p \times q}$, the class $\mathcal{B}$ contains all Borel sets of $p \times q$ real matrices. The theorem is proved.

Definition 2 The $p \times q$ uncertain matrices $\xi_{1}, \xi_{2}, \cdots, \xi_{n}$ are said to be independent iffor any Borel sets $B_{1}, B_{2}, \cdots, B_{n}$ of $p \times q$ real matrices, we have

$$
\mathcal{M}\left\{\bigcap_{i=1}^{n}\left(\xi_{i} \in B_{i}\right)\right\}=\bigwedge_{i=1}^{n} \mathcal{M}\left\{\xi_{i} \in B_{i}\right\}
$$

Example 1 Let $\left(\xi_{i j}\right)_{3 \times 3}$ and $\left(\eta_{i j}\right)_{3 \times 3}$ be independent uncertain matrices. Then, $\left(\xi_{11}, \xi_{12}\right)$ and $\left(\eta_{31}, \eta_{32}, \eta_{33}\right)$ are independent uncertain vectors.

Example 2 Let $\left(\xi_{i j}\right)_{3 \times 3}$ and $\left(\eta_{i j}\right)_{3 \times 3}$ be independent uncertain matrices. Then,

$$
\left(\begin{array}{lll}
\xi_{11} & \xi_{12} & \xi_{13} \\
\xi_{21} & \xi_{22} & \xi_{23}
\end{array}\right) \text { and }\left(\begin{array}{ll}
\eta_{11} & \eta_{12} \\
\eta_{21} & \eta_{22} \\
\eta_{31} & \eta_{32}
\end{array}\right)
$$

are independent uncertain matrices.

Theorem 2 The $p \times q$ uncertain matrices $\xi_{1}, \xi_{2}, \cdots, \xi_{n}$ are independent if and only if

$$
\mathcal{M}\left\{\bigcup_{i=1}^{n}\left(\xi_{i} \in B_{i}\right)\right\}=\bigvee_{i=1}^{n} \mathcal{M}\left\{\xi_{i} \in B_{i}\right\}
$$

for any Borel sets $B_{1}, B_{2}, \cdots, B_{n}$ of $p \times q$ real matrices.

Proof It follows from the duality of uncertain measure that $\xi_{1}, \xi_{2}, \cdots, \xi_{n}$ are independent if and only if

$$
\begin{aligned}
& \mathcal{M}\left\{\bigcup_{i=1}^{n}\left(\xi_{i} \in B_{i}\right)\right\}=1-\mathcal{M}\left\{\bigcap_{i=1}^{n}\left(\xi_{i} \in B_{i}^{c}\right)\right\} \\
& =1-\bigwedge_{i=1}^{n} \mathcal{M}\left\{\xi_{i} \in B_{i}^{c}\right\}=\bigvee_{i=1}^{n} \mathcal{M}\left\{\xi_{i} \in B_{i}\right\} .
\end{aligned}
$$

The theorem is thus proved.

Theorem 3 Let $\xi_{1}, \xi_{2}, \cdots, \xi_{n}$ be independent uncertain matrices, and let $f_{1}, f_{2}, \cdots, f_{n}$ be matrix-valued measurable functions. Then, $f_{1}\left(\xi_{1}\right), f_{2}\left(\xi_{2}\right), \cdots, f_{n}\left(\xi_{n}\right)$ are also independent uncertain matrices. 
Proof For any Borel sets $B_{1}, B_{2}, \cdots, B_{n}$ of real matrices, it follows from the definition of independence that

$$
\begin{aligned}
& \mathcal{M}\left\{\bigcap_{i=1}^{n}\left(f_{i}\left(\xi_{i}\right) \in B_{i}\right)\right\}=\mathcal{M}\left\{\bigcap_{i=1}^{n}\left(\xi_{i} \in f_{i}^{-1}\left(B_{i}\right)\right)\right\} \\
& =\bigwedge_{i=1}^{n} \mathcal{M}\left\{\xi_{i} \in f_{i}^{-1}\left(B_{i}\right)\right\}=\bigwedge_{i=1}^{n} \mathcal{M}\left\{f_{i}\left(\xi_{i}\right) \in B_{i}\right\} .
\end{aligned}
$$

Thus, $f_{1}\left(\xi_{1}\right), f_{2}\left(\xi_{2}\right), \cdots, f_{n}\left(\xi_{n}\right)$ are independent uncertain variables.

\section{Conclusion}

This paper presented a concept of uncertain matrix that is a measurable function from an uncertainty space to the set of real matrices. It was proved that an uncertain matrix is a matrix all of whose elements are uncertain variables. The independence of uncertain matrices was also investigated.

\section{Acknowledgements}

This work was supported by National Natural Science Foundation of China (Grant No.61573210).

\section{Competing interests}

The author declares that he has no competing interests.

Received: 10 October 2016 Accepted: 20 October 2016

Published online: 08 November 2016

\section{References}

1. Liu, B: Uncertainty theory. 2nd ed. Springer, Berlin (2007)

2. Liu, B: Some research problems in uncertainty theory. J. Uncertain. Syst. 3(1), 3-10 (2009)

3. Liu, B: Fuzzy process, hybrid process and uncertain process. J. Uncertain. Syst. 2(1), 3-16 (2008)

4. Liu, B: Uncertain set theory and uncertain inference rule with application to uncertain control. J. Uncertain. Syst. 4(2), 83-98 (2010)

5. Peng, ZX, Iwamura, K: A sufficient and necessary condition of uncertainty distribution. J. Interdiscip. Math. 13(3), 277-285 (2010)

6. Liu, B: Uncertainty theory: a branch of mathematics for modeling human uncertainty. Springer, Berlin (2010)

7. Liu, B: Polyrectangular theorem and independence of uncertain vectors. J. Uncertain. Anal. Appl. 1(9) (2013)

\section{Submit your manuscript to a SpringerOpen ${ }^{\circ}$ journal and benefit from:}

- Convenient online submission

- Rigorous peer review

- Immediate publication on acceptance

Open access: articles freely available online

- High visibility within the field

- Retaining the copyright to your article 